\title{
GAN Dissection and Datacenter RPCs
}

VISUALIZING AND UNDERSTANDING GANS; DATACENTER RPCS CAN BE GENERAL AND FAST.

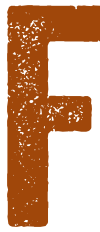

or this edition of "The Morning Paper," I've chosen two papers from very different areas.

Image generation using GANs (generative adversarial networks) has made astonishing progress over the past few years. While staring in wonder at some of the incredible images, it's natural to ask how such feats are possible. "GAN Dissection: Visualizing and Understanding Generative Adversarial Networks" gives us a look under the hood to see what kinds of things are being learned by GAN units, and how manipulating those units can affect the generated images.

February saw the 16th edition of the Usenix Symposium on Networked Systems Design and Implementation. Kalia et al. blew me away with their work on fast RPCs (remote procedure calls) in the datacenter. Through a carefully considered design, they show that RPC performance with commodity CPUs and standard lossy Ethernet can be competitive with specialized systems based on FPGAs (field-programmable gate arrays), programmable switches, and RDMA (remote direct memory access). It's a fabulous reminder to ensure we're making the most of what we already have before leaping to more expensive solutions.

For the complete column, go to https:/lqueue.acm.orgl TheMorningPaper 
Adrian Colyer is a venture partner with Accel in London, where it's his job to help find and build great technology companies across Europe and Israel. IIf you're working on an interesting technology-related business, he would love to hear from you: you can reach him at acolyer@accel.com.] Prior to joining Accel, he spent more than 20 years in technical roles, including CTO at Pivotal, VMware, and SpringSource.

Copyright ( 2019 held by ownerlauthor. Publication rights licensed to ACM. Reprinted with permission from https:I/blog.acolyer.org. 